\title{
EFECTO DE DOS SISTEMAS DE RIEGO SOBRE LA PRODUCCIÓN Y USO EFICIENTE DEL AGUA EN EL CULTIVO DE PAPA VARIEDAD DIACOL CAPIRO
}

\author{
EFFECT OF TWO IRRIGATION SYSTEMS \\ ON PRODUCTION AND EFFICIENT USE OF WATER \\ IN POTATO CROP DIACOL CAPIRO VARIETY
}

\author{
1 Juan Carlos Guerrero Guio \\ 2 Marco Cabezas Gutiérrez \\ ${ }^{3}$ Jesús Hernando Galvis Quintero \\ 1, 2Universidad Pedagógica y Tecnológica de Colombia \\ ${ }^{3}$ Instituto de Educación Técnica Profesional de Roldanillo (INTEP) \\ ${ }^{1}$ juancarlos.guerrero@uptc.edu.co ${ }^{2}$ mcabezasg@unal.edu.co, ${ }^{3}$ jesus.galvis@gmail.com
}

\section{RESUMEN}

La papa (Solanum tuberosum) se considera un alimento básico en la dieta de cientos de personas. En Colombia, durante el 2017 se registró una producción de 2,8 millones de toneladas siendo la variedad Diacol capiro la más sembrada para procesamiento industrial en los departamentos de Cundinamarca, Boyacá y Nariño. El cultivo es sensible al déficit hídrico y es el factor que más limita su producción, por lo tanto, el riego es una práctica indispensable que se está realizando por aspersión con una baja eficiencia mientras el riego por goteo puede alcanzar una eficiencia cercana al $90 \%$. Se realizó un experimento en condiciones de campo cuyo objetivo fue evaluar el efecto de dos sistemas de riego (goteo vs aspersión) sobre la producción, calidad y uso eficiente del agua en el cultivo de papa variedad Diacol capiro en el municipio de Toca, Boyacá. Se usó un diseño experimental completamente aleatorio con 2 tratamientos que corresponden a cada sistema de riego y 3 repeticiones. A los 28 días de siembra (dds) se midió el porcentaje de brotación; en cosecha se evaluó rendimientos y calidad de tubérculos; y se calculó la eficiencia en el uso el agua para tubérculos frescos. Se presentaron diferencias estadísticas para los tratamientos evaluados $(p<0,05)$ en el porcentaje de brotación y uso eficiente del agua; las demás variables no presentaron diferencias estadísticas. Con los dos tratamientos se alcanzó el potencial productivo de la variedad, pero el riego por goteo presentó los mejores resultados dado que se llegó a producir 3,49 kg más de tubérculos frescos por $\mathrm{m} 3$ de agua utilizado.

Palabras clave: eficiencia en el uso del agua; riego por aspersión; riego por goteo; rendimiento de cultivos; Solanum tuberosum 


\section{ABSTRACT}

Potato (Solanum tuberosum) is considered a staple in the diet of hundreds of people. In Colombia, during 2017, a production of 2.8 million tons was registered, being the variety Diacol Capiro most planted for industrial processing in the departments of Cundinamarca, Boyacá and Nariño. The crop is sensitive to water deficit and it is the most limiting factor in production, therefore, irrigation is an essential practice which is being done by spraying with low efficiency, while drip irrigation can achieve efficiencies close to $90 \%$. An experiment was carried out under field conditions aiming to evaluate the effect of two irrigation systems (drip vs spray) on the production, quality, and efficient use of water in the Diacol Capiro variety potato crop in the municipality of Toca, Boyacá. A completely randomized experimental design was used with 2 treatments corresponding to each irrigation system and 3 repetitions. At 28 days after sowing (das) sprouting percentage, yield and quality of tubers were measured and harvest and efficient water use was calculated for fresh tubers. Statistical differences were observed for the evaluated treatments $(p<0.05)$ in germination and efficient use of water; other variables did not show statistical differences. With two treatments the productive potential of the variety was reached but the drip irrigation presented the best results, producing an additional $3.49 \mathrm{~kg}$ of fresh tubers per $\mathrm{m} 3$ of water used.

Keywords: cropyield; drip irrigation; sprinkler irrigation; Solanum tuberosum; water use efficiency.

\section{INTRODUCCIÓN}

La papa (Solanum tuberosum L.) se considera un alimento básico por sus altos contenidos nutricionales; un importante proveedor de carbohidratos, vitaminas y minerales en las dietas de cientos de personas en todo el mundo (Camire, 2016); y es el tercer cultivo más importante después del trigo y el maíz. En el 2017, a nivel global se produjeron 388 millones de toneladas en 20 millones de hectáreas y los principales productores durante ese año fueron China, India, Rusia, Ucrania y Estados Unidos (FAOSTAT 2017).

En el año 2017, Colombia registró un área de siembra de 169.000ha, una producción de 2,8 millones de toneladas con un rendimiento promedio nacional de 16,9t/ha-1. La variedad más sembrada para procesamiento industrial es la Diacol capiro conocida comercialmente como $\mathrm{R}-12$ : esta tiene un rendimiento potencial por encima de 40 t.ha $^{-1}$, presenta alta aceptación para procesamiento industrial tanto en hojuela como en bastón por tener un color de carne crema claro y buena respuesta a la fritura, además de una buena aceptación para consumo en fresco (Porras y Herrera, 2015; Ñústez, 2011).

El cultivo es sensible al déficit hídrico (Dalla y Mackerron, 2006; Kafkafi y Trchitzky, 2012; Quiroz, 2012) y la escasez de agua provoca una reducción en la producción de tubérculos (Rodríguez-Perez, 2010; Quiroz, 2012). Son muchos los autores a nivel mundial que han realizado estudios para relacionar el suministro de agua con la productividad, entre ellos: Zhou et al, (2018) en Dinamarca; Zin El-Abedin et al (2017) en Arabia Saudita; Yang et al (2017) en China; Reyes-Cabrera et al (2016) en Estados Unidos; Peña y Zenner (2015) en Colombia; Rodríguez et al (2015) en Argentina; 
Hess et al (2015) en Gran Bretaña y Shahnazari et al (2007) en Dinamarca.

Para alcanzar los rendimientos potenciales, el cultivo tiene un requerimiento hídrico de entre 400 a 700 mm (Manickam et al, 2018; Quiroz, 2012) y la productividad se ve afectada si se reduce más del $30 \%$ del agua disponible en el suelo (Rodríguez- Pérez, 2010). Al respecto la FAO (2008) sugiere que la productividad se ve afectada si se reduce más del $50 \%$ del agua disponible en el suelo. La etapa más crítica y de mayor exigencia de agua es la floración, que coincide con la tuberización (100 dds), pues su disponibilidad limitada reduce el número de tubérculos por planta. (Corpoica, 2009, Manickam et al, 2018).

De acuerdo a lo anterior, el riego es una práctica indispensable que se debe realizar para alcanzar el potencial productivo del cultivo en las zonas productoras de bajas precipitaciones; esta se realiza mediante sistema de riego convencional de aspersión, pero la FAO (2002) argumenta que el riego de superficie es menos eficiente y en ocasiones causa problemas de anegamiento y salinización.

El sistema de riego por goteo se ha implementado en diversos cultivos, tiene una eficiencia del $90 \%$ al permitir la entrega directa de agua al punto que la planta lo demanda (Kafkafi y tarchitzky, 2012). Según FAO (2002), los resultados obtenidos en varios países demuestran que el cambo de riego por surcos o riego por aspersión, a riego por goteo puede reducir el consumo de agua entre $30 \%$ y $60 \%$. Al respecto, Chawla y Singh (2016) afirman que con riego por goteo se puede ahorrar un $46 \%$ de agua con respecto al riego por gravedad y en definitiva el riego por goteo es eficiente y representa un avance tecnológico con amplio rango de aplicación.

Considerando lo anterior, la hipótesis de esta investigación es que el potencial productivo del cultivo de papa variedad Diacol capiro se puede alcanzar con una mayor eficiencia en el uso del agua. El objetivo de la investigación es comparar los dos sistemas de riego, riego por goteo y riego por aspersión, en términos de la brotación, producción y calidad de tubérculos y productividad del uso de agua en el cultivo de papa variedad Diacol capiro.

\section{MATERIALES Y MÉTODOS}

La investigación, se llevó a cabo durante el primer semestre de 2018 en un lote comercial perteneciente a la Sociedad de Pequeñas Familias Productoras de Papa Industrial Asoagrotoca, ubicado en la finca La Planada (Vereda de Cunucá, municipio de Toca, Boyacá) con coordenadas $50^{\circ} 36^{\prime} 14^{\prime \prime} \mathrm{N} 73^{\circ}$ 09' 23" O, a una altura de 2810 m.s.n.m y una temperatura promedio de $12^{\circ} \mathrm{C}$. La zona presenta una precipitación anual de $800 \mathrm{~mm}$ de régimen bimodal distribuidos de marzo a mayo y de septiembre a noviembre.

El suelo seleccionado para la ubicación del ensayo fue clasificado como Inceptisol, que presenta un relieve plano con pendientes del $1 \%$, y características físicas y químicas homogéneas. La tabla I describe las características químicas iniciales de suelo, la gráfica II y III describen las características físicas iniciales a la profundidad de $0-20 \mathrm{~cm}$ y $20-$ $40 \mathrm{~cm}$ respectivamente. 
Tabla 1. características químicas iniciales del suelo

\begin{tabular}{|c|c|c|c|c|c|c|c|c|c|c|c|c|c|c|}
\hline \multicolumn{4}{|c|}{ Acidez } & \multicolumn{4}{|c|}{$\begin{array}{c}\text { Bases intercambiables } \\
\left(\mathrm{Cmol}^{\mathrm{kgg}}{ }^{-1}\right)\end{array}$} & \multirow[t]{2}{*}{$\begin{array}{l}\% \\
\text { MO }\end{array}$} & \multirow[t]{2}{*}{$\begin{array}{c}\mathbf{P} \\
(\mathbf{m g} \\
\left.\mathbf{k g}^{-1}\right)\end{array}$} & \multicolumn{5}{|c|}{$\begin{array}{c}\text { Elementos menores } \\
\qquad\left(\mathbf{m g} \cdot \mathbf{k g}^{-1}\right)\end{array}$} \\
\hline $\mathrm{pH}$ & $\begin{array}{l}\text { Ac, Inter. } \\
(\mathrm{Cmol.Kg})\end{array}$ & $\% \mathrm{Al}^{3+}$ & $\mathrm{Ca}$ & $\mathrm{Mg}$ & $\mathrm{K}$ & $\mathrm{Na}$ & $\mathrm{Ca}+\mathrm{Mg}+\mathrm{K}$ & & & $\mathrm{Fe}$ & $\mathrm{Mn}$ & $\mathrm{Cu}$ & $\mathrm{Zn}$ & B \\
\hline 5,4 & 0,11 & 1,7 & 4,5 & 0,8 & 0,81 & 0,24 & 6,11 & 3,41 & 30 & 454 & 8,3 & 8,9 & 0,4 & 0,05 \\
\hline
\end{tabular}

Tabla 2. características físicas iniciales del suelo a profundidad de $0-20 \mathrm{~cm}$

\begin{tabular}{|c|c|c|c|c|c|c|c|c|c|}
\hline \multicolumn{3}{|c|}{ textura } & \multirow{2}{*}{$\begin{array}{c}\text { Densidad } \\
\text { aparente } \\
\text { g. } \mathrm{Cc}^{-1}\end{array}$} & \multirow{2}{*}{$\begin{array}{c}\text { Densidad } \\
\text { real } \\
\text { g. } \mathrm{Cc}^{-1}\end{array}$} & \multirow{2}{*}{$\begin{array}{c}\text { Porosidad } \\
\text { total } \\
\qquad \%\end{array}$} & \multirow{2}{*}{$\begin{array}{c}\text { Macroporos } \\
\%\end{array}$} & \multirow{2}{*}{$\begin{array}{c}\text { Mesoporos } \\
\%\end{array}$} & \multirow{2}{*}{$\begin{array}{c}\text { Microporos } \\
\text { \% }\end{array}$} & \multirow{2}{*}{$\begin{array}{c}\text { Conductividad } \\
\text { Hidráulica } \\
\text { saturada } \\
\text { cm. } \mathrm{h}^{-1}\end{array}$} \\
\hline$\% A$ & $\% \mathrm{~L}$ & $\% \mathrm{Ar}$ & & & & & & & \\
\hline 24,3 & 29 & 46,7 & 0,97 & 2,39 & 59,47 & 34,50 & 12,99 & 11,98 & 23,78 \\
\hline
\end{tabular}

Tabla 3. características físicas iniciales del suelo a profundidad de $20-40 \mathrm{~cm}$

\begin{tabular}{c|c|c|c|c|c|c|c|c|c|}
\multicolumn{2}{|c|}{ textura } & $\begin{array}{c}\text { Densidad } \\
\text { aparente }\end{array}$ & $\begin{array}{c}\text { Densidad } \\
\text { real }\end{array}$ & $\begin{array}{c}\text { Porosidad } \\
\text { total }\end{array}$ & Macroporos & Mesoporos & $\begin{array}{c}\text { Microporos } \\
\text { Hidráulica } \\
\text { saturada }\end{array}$ \\
\hline$\% \mathrm{~A}$ & $\% \mathrm{~L}$ & $\% \mathrm{Ar}$ & $\mathrm{g} \cdot \mathrm{Cc}^{-1}$ & $\mathrm{~g} \cdot \mathrm{Cc}^{-1}$ & $\%$ & $\%$ & $\%$ & $\%$ \\
\hline 31,2 & 29.4 & 39,1 & 1,08 & 2,36 & 54,11 & 23,06 & 8,41 & 22,64 \\
\hline
\end{tabular}

Se utilizaron semillas de papa certificada de la variedad Diacol capiro, con un diámetro entre 7 a $10 \mathrm{~cm}$. El trabajo se realizó bajo condiciones de campo; primero se realizó la preparación del terreno a una profundidad de $40 \mathrm{~cm}$, luego se llevó a cabo la siembra ubicando el fertilizante en la base del surco en las siguientes cantidades: $140 \mathrm{~kg} / \mathrm{ha}^{-1}$ $\left(\mathrm{NO}_{3}\right), 145 \mathrm{~kg} / \mathrm{ha}^{-1}\left(\mathrm{NH}_{4}\right), 242 \mathrm{~kg} / \mathrm{ha}^{-1}$ (P2O5), $325 \mathrm{~kg} / \mathrm{ha}_{-1}\left(\mathrm{~K}_{2} \mathrm{O}\right), 91 \mathrm{~kg} / \mathrm{ha}^{-1}$ (C2O), 54kg/ $\mathrm{ha}^{-1}$ (MgO), $90 \mathrm{~kg} / \mathrm{ha}^{-1}$ (SO4), 0,7kg/ha-1 (Fe), $2,1 \mathrm{~kg} / \mathrm{ha}^{-1}$ (B), $0,07 \mathrm{~kg} / \mathrm{ha}^{-1}$ (Mn), 0,97kg/ha-1 $(\mathrm{Zn})$. Luego, se colocó una semilla por sitio a $10 \mathrm{~cm}$ de profundidad. Después, se realizó la respectiva desinfección de la semilla y la base del surco (se aplicó Azoxistrobina y
Difenoconazole, Permetrina) y se tapó formando un surco alto donde posteriormente se ubicó la manguera de goteo. El plan de fertilización se definió de acuerdo a las características químicas iniciales del suelo y las tablas de extracción tomadas de Castro y Gómez (2013).Se realizó el respectivo control de plagas y enfermedades. A los 60 DDS se realizó la labor de aporque. Se empleó un diseño experimental completamente al azar (DCA) con 2 tratamientos y 3 repeticiones para un total de 6 unidades experimentales (UE). Cada UE medía $12 \mathrm{~m}$ de ancho y $40 \mathrm{~m}$ de largo. La distancia de siembra utilizada fue de $1 \mathrm{~m}$ entre surcos y $0,4 \mathrm{~m}$ entre plantas para una población de 25.000 plantas ha-1 
Los tratamientos fueron T1, Riego por goteo, y T2, Riego por aspersión. Para el sistema de riego por goteo se utilizó manguera de goteo UniRam ${ }^{\mathrm{TM}}$ AS marca Netafin $\AA$, auto compensada, con goteros a $10 \mathrm{~cm}$ y con una descarga de $1 \mathrm{l} / \mathrm{ha}^{-1}$; se instaló en el momento de la siembra sobre el surco y en el momento de realizar el aporque (50 días después de la siembra, dds) se enterró a una profundidad de $10 \mathrm{~cm}$. Para el sistema de riego por aspersión, se usaron aspersores Xcel-Wobbler marca Senninger $\AA$, boquilla \#14 azul(5.56 mm) con una descarga de $1.583 \mathrm{l} / \mathrm{ha}^{-1}$; que se instaló a una distancia de $6 \mathrm{~m}$ entre aspersores en la línea media de la unidad experimental. 25dds se registró el porcentaje de emergencia de cada unidad experimental.

En el momento de la cosecha se realizaron 3 muestreos por UE, de cada muestreo se seleccionó la papa primera (diámetro 5-10 cm); papa segunda (diámetro 3-5 cm); papa riche (diámetro $<3 \mathrm{~cm}$ ); papa verde y papa con daño fitosanitario causado por Tecia sonalivora (Povolny); estos datos se expresan en t/ha ${ }^{-1}$. Para evaluar la cantidad de agua requerida por el cultivo, por cada UE se elaboró una curva de retención de humedad a 4 puntos $(0 ; 0,3 ; 1$ y 15 Bares) y de acuerdo a estas se calculó el agua disponible para la planta en el suelo (ADPS) (agua retenida a una presión de entre 0,3 y 15 bares) en porcentaje de humedad volumétrico. Los programas de riego se fijaron de acuerdo al agotamiento del ADPS, para esto se fijó un porcentaje de agotamiento por estado fenológico (Tabla IV). Según la FAO (2008) y Rodríguez-Pérez (2010), el estado fenológico de floración corresponde al mayor consumo de agua pues presenta la mayor actividad fotosintética. De acuerdo a lo anterior, en dicho estado fenológico se fijó el punto de agotamiento más bajo y en los demás estados fenológicos se fijó un punto de agotamiento más alto. Para esto, se contó con un kit sensor de humedad de suelo SM150T que permitió monitorear la humedad en campo; por cada UE se realizaron monitoreos 2 veces por semana en 3 sitios diferentes, en cada sitio se elaboró un perfil de $40 \mathrm{~cm}$ de profundidad. Desde el dds 0 al 60, se midió a una profundidad entre $0-20 \mathrm{~cm}$ y desde el día $60 \mathrm{dds}$ (realizo del aporque) se midió la humedad a la profundidad entre $0-20 \mathrm{~cm}$ y entre 20 a $40 \mathrm{~cm}$.

Tabla 5. Porcentaje de agotamiento permitido por estado fenológico para el cultivo de papa variedad Diacol Capiro según ADPS.

\begin{tabular}{l|c} 
Estado fenológico & $\begin{array}{c}\text { Porcentaje de agotamiento } \\
\text { permitido según ADPS* }\end{array}$ \\
\hline Brotación-Germinación & $75 \%$ \\
\hline Vegetativo & $75 \%$ \\
\hline Floración & $50 \%$ \\
Senescencia & $75 \%$ \\
\hline *ADPS: agua disponible para la planta en el suelo & \\
\hline
\end{tabular}

Fuente: adaptado de FAO, 2008; Rodríguez- Pérez, 2010.

La precipitación se midió con un pluviómetro instalado en la finca. 
Se calculó el uso eficiente del agua para rendimientos de tubérculos frescos (cantidad de tubérculo producido por $\mathrm{m}^{3}$ de agua utilizado), utilizando la siguiente relación:

$$
\text { Uso efieciente del agua }=\frac{\text { (Produccion de tubérculo fresco }(\mathrm{kg})}{\text { (Agua aplicada }(\mathrm{m} 3)}
$$

Los datos obtenidos en cada variable fueron sometidos a pruebas de normalidad mediante el método de Shapiro-Will y de homogeneidad de varianzas mediante la prueba de Levene. Comprobados los supuestos, se realizó la prueba de T-Student para muestras independientes $(P \leq 0,05)$ con el fin de saber si existían diferencias entre las medias de los dos tratamientos para cada variable. Los análisis se realizaron con el programa estadístico SPSS 25v del año 2017.

\section{RESULTADOS Y DISCUSIÓN}

\section{Porcentaje de brotación}

La brotación a los 25 dds presentó diferencias estadísticas significativas ( $p \leq 0,05$ ). La mejor brotación se presentó en el tratamiento de riego por goteo con $71,66 \pm 4,04 \%$ mientras que en el tratamiento de riego por aspersión fue de 23,33 $\pm 2,02 \%$; se necesitaron $11 \mathrm{~mm}$ y $43 \mathrm{~mm}$ de agua respectivamente para tener la brotación en cada uno de los sistemas. (Figura 1)

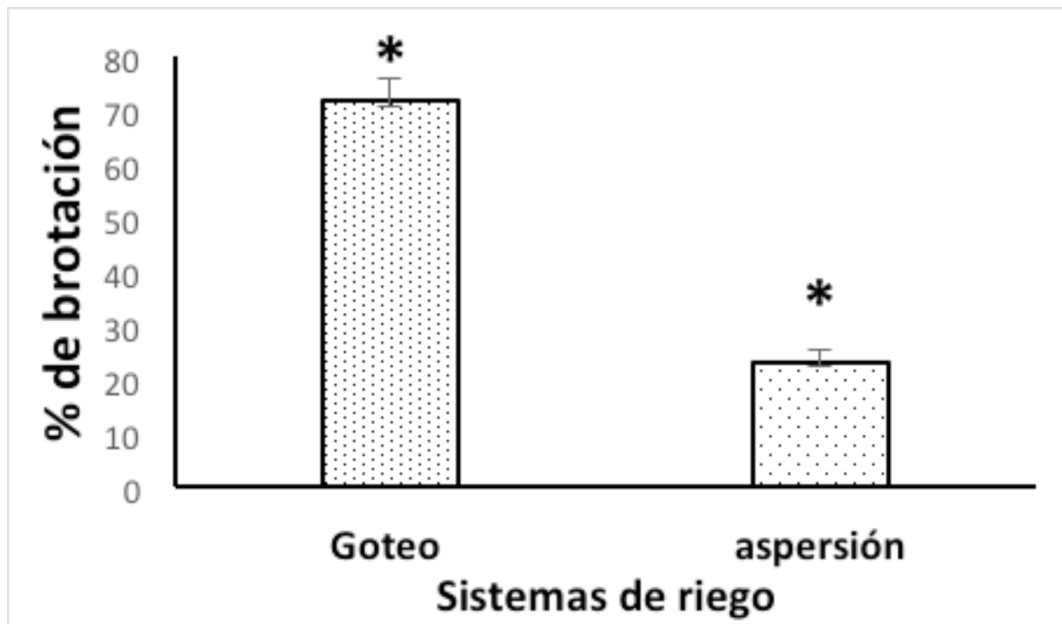

Figura 1. Efecto de dos sistemas de riego sobre la brotación de tubérculos de papa. Según la prueba de T-Student $(P \leq 0,05)$, ns: no existen diferencia estadística, *: diferencias significativas.

Los contenidos de humedad durante los primeros $25 \mathrm{dds}$ a $20 \mathrm{~cm}$ de profundidad fueron del $29 \%$ y $27 \%$ para riego por goteo y riego por aspersión respectivamente. El mayor contenido de humedad en el sistema de goteo se debe a la distribución del agua dentro del suelo de cada sistema. En el sistema de riego por aspersión (sistema tradicional de la zona) el movimiento de agua sigue un patrón de percolación vertical unidimensional en donde solo la fuerza gravitacional dirige el movimiento del agua hacia abajo, mientras que en el sistema de riego por goteo el agua dentro del suelo sigue un patrón de distribución tridimensional en que hay dos fuerzas que afectan simultáneamente el flujo de agua: gravitacional y de capilaridad, que impulsan el agua en todas las direcciones(Kafkafi y tarchitzky, 2012) 
inclusive hacia arriba, permitiendo una mejor retención de agua en el suelo y una mayor disponibilidad para la planta.

Al respecto, Quiroz (2012) dice que la brotación de tubérculos puede retardarse por la humedad limitada del suelo; en este caso no se presentó humedad limitada, al contrario hubo una mayor disponibilidad de agua en el sistema de riego por goteo que permitió acelerar la brotación con respecto al riego por aspersión.

\section{Rendimiento y calidad de tubérculos frescos}

Ninguna de las variables evaluadas en el momento de la cosecha (rendimiento total, rendimiento de los tamaños de papa primera, papa segunda, papa riche, papa con daños por insecto y papa verde) presentó diferencias estadísticas significativas $(p<0,05)$ (Figura 2).

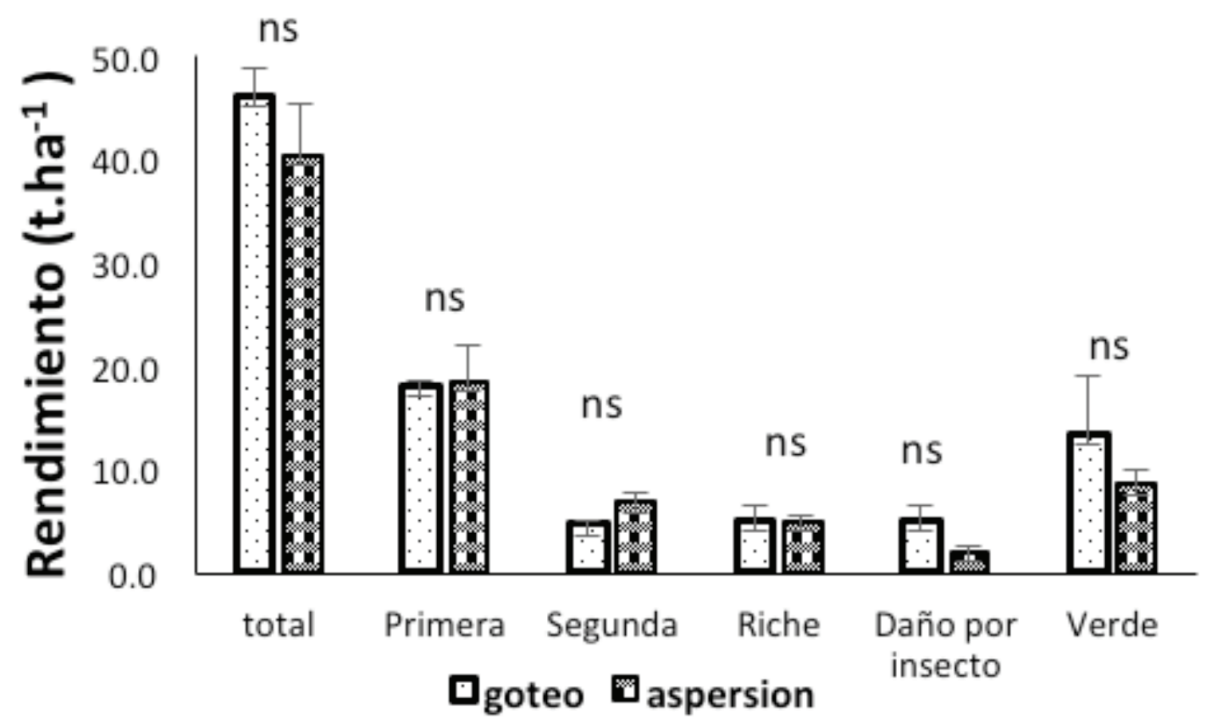

Figura 2. Efecto de dos sistemas de riego sobre el rendimiento y la distribución de tamaños de tubérculos de papa. Según la prueba de T-Student $(P \leq 0,05)$, ns: no existen diferencia estadística, *: diferencias significativas.

El rendimiento estimado para el tratamiento de riego por goteo fue de $46,1 \pm 2,5 \mathrm{t} /$ $\mathrm{ha}^{-1}$ mientras que en riego por aspersión fue de 40,3 3 4,8 t/ha-1. Los dos tratamientos presentan rendimientos más altos del potencial productivo de la variedad el cual está 40 t/ha-1 según Ñústes (2011); Porras y Herrera (2015). Sin embargo, el tratamiento de riego por goteo presentó $5,8 \mathrm{t} / \mathrm{ha}^{-1}$ más en comparación al tratamiento de riego por aspersión: cantidad que no es significativa estadísticamente, pero agronómicamente un diferencial de $5,8 \mathrm{t} / \mathrm{ha}^{-1}$ si es significativo. Peña y Zenner (2015), evaluaron la respuesta de la papa variedad Diacol capiro a la aplicación de riego y encontraron un aumento del $18 \%$ en el rendimiento con la aplicación de riego por goteo con respecto a un tratamiento sin aplicación de riego en una zona de precipitaciones homogéneas, se obtuvieron resultados similares a los de este trabajo; con el tratamiento de riego por goteo se aumentó el rendimiento en un $14 \%$ con respecto al tratamiento de riego por aspersión. Zhou et al, (2018) también encontraron resultados similares en la variedad folva, similar a la variedad de estudio, concluyendo que no existen diferencias en producción entre la irrigación por goteo versus la 
irrigación superficial con cañón, sin embargo, con la irrigación por goteo encontraron una mayor eficiencia agronómica del uso del nitrógeno durante uno de los años.

Al referirse al rendimiento según los tamaños, en riego por goteo tenemos $18 \pm 0,4 \mathrm{t} / \mathrm{ha}^{-1}$ para papa primera; 4,6 $\pm 0,24 \mathrm{t} / \mathrm{ha}^{-1}$ para papa segunda y $4,9 \pm 1,24 \mathrm{t} / \mathrm{ha}^{-1}$ para papa riche. Mientras que en riego por aspersión encontramos 18,3 $\pm 3,4 \mathrm{t} / \mathrm{ha}^{-1}$ para papa primera; 6,6 $\pm 0,9 \mathrm{t} / \mathrm{ha}^{-1}$ para papa segunda y $4,8 \pm 0,35 \mathrm{t} / \mathrm{ha}^{-1}$ para papa riche. Se presentan rendimientos muy similares en los tres tamaños para los dos sistemas de riego. Camire (2016) obtuvieron resultados similares al comparar riego por goteo con riego por gravedad; encontrando menores rendimientos, pero una distribución homogénea en tamaños.

Ferreira et al (2017), en la variedad Atlantic, encontró rendimientos similares en tubérculos totales y comercializables a pesar de tener en un tratamiento de mayor desarrollo de raíces.

En cuanto a problemas de tubérculos no aptos para el consumo humano, se presentaron daños por insecto de Tecia solanivora (Povolny) y papa verde. En riego por goteo estos daños fueron de $5 \pm 1,4 \mathrm{t} / \mathrm{ha}^{-1}$ para daño por insecto $\mathrm{y}$ $13,3 \pm 5,4 \mathrm{t} / \mathrm{ha}^{-1}$ para papa verde mientras en riego por aspersión fueron de 1,8 $\pm 0,41$ t/ha ${ }^{-1}$ para daño por insecto y $8,4 \pm 1,3 \mathrm{t} / \mathrm{ha}^{-1}$ para papa verde (figura 2). A pesar de no presentarse diferencias significativas, es evidente un mayor daño de $T$ solanivora y papa verde en el sistema de riego por goteo.

Actualmente no existen trabajos que relacionen los daños de $T$ solanivora y papa verde con los diferentes sistemas de riego: estos daños se deben al área de mojado de la superficie del suelo en cada sistema de riego evaluado. Reyes-Cabrera et at (2014), en Estados Unidos, encontraron que con la irrigación por goteo había una reducción significativa de la incidencia de trastornos fisiológicos de los tubérculos como el centro marrón, el corazón hueco y la necrosis por calor interno.

En el sistema de riego por aspersión, el riego es superficial y tiene un cubrimiento de humedecimiento del $100 \%$ de la superficie del suelo por lo que mantuvo contenidos de humedad a capacidad de campo y se evitó la formación de grietas mientras que en el sistema de riego por goteo enterrado, el riego no tuvo un cubrimiento total de humedecimiento de la superficie y esta mantuvo contenidos de humedad por debajo de capacidad de campo generando la formación de grietas. Según Jaramillo (2014), esto sucede en suelos con medios contenidos de arcilla, como es el caso del suelo en estudio (contenidos de arcilla mayor al 25\%). Cuando las arcillas se secan se genera una contracción y una reducción del volumen ocupado generando la formación de grietas; dichas grietas sirvieron como vía para que las larvas de $T$ solanivora accedieran a los tubérculos y causaran el daño, además, para que los rayos solares alcanzaran alguna parte del tubérculo y causar el verdeamiento.

Al respecto, Yang et at (2017) encontraron un uso más eficiente del agua con una proporción de suelo húmedo de $40 \%$ contra el $70 \%$, con utilización de plástico como mulch; que en la zona de estudio funciona como una barrera para el ataque de $T$ solanivora y bloquea los rayos solares. Zhang et al (2017) encontró que un $35 \%$ de porcentaje de humedecimiento más la cobertura plástica trasparente y el riego por goteo son una buena técnica que amerita más pruebas para el noroeste árido de China.

Kafkafi y tarchitzky (2012) afirman que la ubicación profunda de la cinta derivó en una reducción del rendimiento y del grado comercial debido a varias razones: pobre suministro de agua al sistema radicular somero de las papas, 
deficiencias de humedecimiento en la epidermis externa de los tubérculos, y sobrecalentamiento del tubérculo en el lomo relativamente seco (papa verde).

Un aspecto a resaltar que presenta el sistema de aspersión sobre el daño de $T$ solanivora, es el agua libre en el suelo en el momento del riego, la cual se presenta cuando el suministro de agua supera la velocidad de infiltración. Según SIPSA (2014) el estado larval de $T$ solanivora corresponde al estado donde la plaga hace el daño sobre los tubérculos y es el estado donde es más susceptible al agua.

\section{Uso eficiente del agua}

El uso eficiente del agua ( $\mathrm{Kg}$ tubérculos fres$\cos \times \mathrm{m}^{-3}$ de agua) presentó diferencias estadísticas significativas $(p<0,05)$. En el sistema de riego por goteo se presentó un rendimiento de $8,8 \pm 0,48 \mathrm{~kg}$ tubérculos frescos $/ \mathrm{m}^{-3} \mathrm{de}$ agua; mientras que en riego por aspersión el rendimiento fue de 5,31 $\pm 0,64 \mathrm{~kg}$ tubérculos frescos $/ \mathrm{m}^{-3}$ de agua. En el sistema de riego por goteo se produjeron $46,1 \mathrm{t} / \mathrm{ha}^{-1}$ con tan solo $521 \mathrm{~mm}$ contra 40,3 t/ha-1 con $759 \mathrm{~mm}$ en el sistema de riego por aspersión (figura 3).

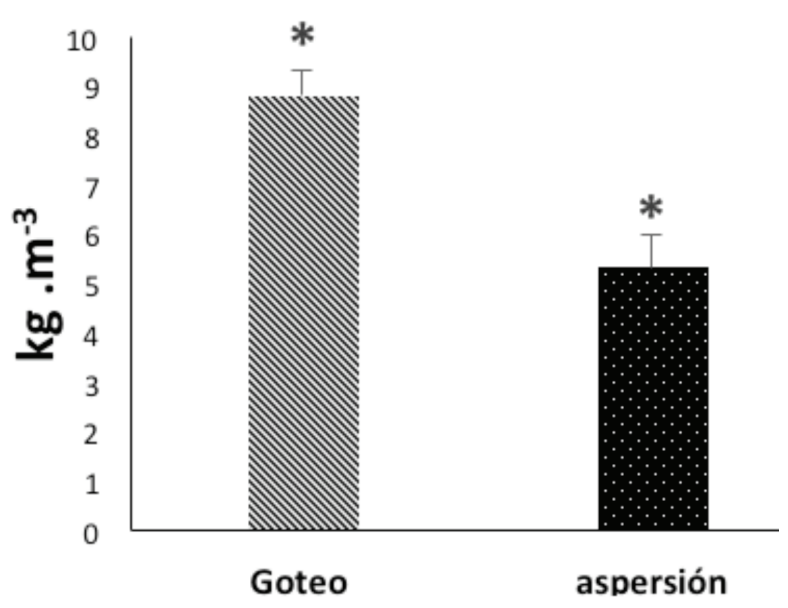

Figura 3. Uso eficiente del agua en el cultivo de papa por efectos de dos sistemas de riego. Según la prueba de T-Student $(P \leq 0,05)$, ns: no existen diferencias estadísticas significativas.

Son varios los estudios realizados que han determinado el consumo de agua de un cultivo de papa; al respecto, la FAO en el año internacional de la papa en el 2008 declara que un cultivo de papa de 120 a 150 días requiere de $500 \mathrm{~mm}$ a $700 \mathrm{~mm}$ de agua. La misma FAO, en el 2012, declaró que los consumos de agua a campo abierto son de $350 \mathrm{~mm}$ a $650 \mathrm{~mm}, \mathrm{y}$ otros autores alrededor del mundo citan valores similares entre ellos Rodríguez et al, 2015. Al relacionar esto con el presente estudio encontramos que el consumo de agua en el sistema de riego por goteo está dentro de los valores citados, muy cercano al límite inferior, mientras que el sistema de riego por aspersión esta fuera del rango. De acuerdo a lo anterior, sería lógico afirmar que para mantener los contenidos adecuados de humedad en el suelo para el cultivo de papa utilizando el sistema de riego por aspersión, se debe tener una oferta hídrica mayor a $700 \mathrm{~mm}$.

En cuanto al uso eficiente del agua para tubérculos frescos, Quiroz (2012) reporta un valor de 4 a $11 \mathrm{~kg} / \mathrm{m}^{-3}$ de agua, los valores obtenidos en el presente estudio están dentro de este rango para los dos sistemas de riego utilizados, aunque se llegaron a producir 3,49 kg más de tubérculos frescos $/ \mathrm{m}^{-3}$ de agua en el sistema de riego por goteo. Kifle y Gebretsadikan (2016), obtuvieron 
resultados similares al realizar un trabajo con el cultivo de papa en una zona semiárida del Norte de Etiopia y encontrando una productividad del agua de $2,79 \mathrm{~kg} / \mathrm{m}^{-3}$ sin exponer las plantas a estrés hídrico y con un consumo de $673,3 \mathrm{~mm}$. Reyes-Cabrera et al (2016), encontraron una diferencia de $5,7 \mathrm{~kg} / \mathrm{m}^{-3}$ en las variedades Atlantic y Fabula al comparar riego por goteo superficial contra riego por infiltración. Yang et al (2017) obtuvieron un uso más eficiente del agua con riego por goteo y uso de plástico como Mulch a una proporción de humedecimiento del $40 \%$. Nouri et al (2019) encontraron un ahorro del 5\% de agua con la combinación de riego por goteo con mulch orgánico en el suelo.

\section{CONCLUSIONES}

Con el sistema de riego por goteo se obtuvo la mejor brotación de tubérculos a los 28 días después de la siembra y la producción total fue mejor. Esto, estadísticamente no fue significativo, pero agronómicamente las diferencias encontradas son significativas.

En el sistema de riego por goteo la calidad de los tubérculos fue menor a los de riego por aspersión, dado que los tubérculos se vieron afectados en termino de papa verde y la plaga $T$ solanivora.

El uso más eficiente del agua se presentó con el sistema de riego por goteo (30\% menos de agua requerida) aunque las producciones totales fueran similares.

\section{LITERATURA CITADA}

Camire, M. (2016). Potatoes and Human Health. En: Singh, J.; Kaur, L. (eds). Advances in Potato Chemistry and Technology. Elsevier Inc, p, 685-704.

Castro, H. y Gómez, M. (2013). Capítulo 4. Fertilidad de suelos y fertilizantes. En: Burbano, O. y Silva, M. (eds). Ciencia del suelo principios básicos. Primera edición. Bogotá DC:Editorial Guadalupe S.A. p, 139-304.

Chawla, A., Singh, K. (2016). Performance of drip irrigated potato. En: Singh, K.; Goyal, M.; Rudra,
R. (eds). Best Management Practices for Drip Irrigation Crops. Ed Apple Academic Press, Inc, p 299-332.

Corporación Colombiana de Investigación Agropecuaria. (CORPOICA) (2009). Escalas fenológicas de las variedades de papa parda pastusa, Diacol capiro y criolla "yema de huevo" en las zonas productoras de Cundinamarca, Boyacá, Nariño y Antioquia. 34p. Recuperado de: https://repository.agrosavia.co/bitstream/ handle/20.500.12324/12893/44240_56518. pdf? sequence $=1$ \&isAllowed $=y$.

Dalla, L., MacKerron, D (2006). Plant and soil water status. En: Haverkort, A.; MacKerron, D. (eds). Management of nitrogen and water in potato production. Ed. Wageningen Academic Publihers, p,111-173.

Ferreira, C., Zotarelli, L., Tormena, C., Rens, L., Rowland, D. (2017). Effects of water table management on least limiting water range andpotato root growth. Agricultural Water Management, (186), 1-11. Doi: http://dx.doi.org/10.1016/j. agwat.2017.02.020.

FOASTAT (2017). Cantidades de producción de patatas y patatas por país. Recuperado de: http:// www.fao.org/faostat/es/\#data/QC/visualize

Hess, T., Lennard, A., Daccache, A. (2015). Comparing local and global water scarcity information in determining the water scarcity footprint of potato cultivation in Great Britain. Journal of Cleaner Production, (87), 666-674. Doi: http:// dx.doi.org/10.1016/j.jclepro.2014.10.075.

Jaramillo, D. (2014). El suelo: Origen, propidades, especialidad. Universidad Nacional de Colombia. Facultad de ciencias, p, 553.

Kafkafi, U., Tarchitzky, J. (2012). Fertirrigación, una herramienta para una eficiente fertilización y manejo del agua. Asociación Internacional de la Industria de Fertilizantes (IFA) e Instituto Internacional de la Potasa, p 151. https://www. ipipotash.org/udocs/391-2012_ifa_ipi_fertirrigacion.pdf.

Kifle, M., Gebretsadikan, T. (2016). Yield and water use efficiency of furrow irrigated potato ander regulated deficit irrigation, Atsibi-Wemberta, North Ethiopia. Rev. Agricultural Water Management, (170), 133-139. Doi: http://dx.doi.org/10.1016/j. agwat.2016.01.003.

Manickam, J., Pillai, S., Manickam, R. (2018). Water requirements for horticultural crops under micro 
irrigation. En: Goyal, M.; Aladakatti. (eds). Engineering interventions in sustainable trickle irrigation. Ed. Apple Academic Press, Inc, p 3-34.

Nouri, H., Stokvis, B., Galindo, A., Blatchford, M., Hoekstra, A. (2019). Water scarcity alleviation through water footprint reduction in agriculture: The effect of soil mulching and drip irrigation. Science of the Total Environment, (653), 241-252. https://doi.org/10.1016/j.scitotenv.2018.10.311.

Nústez, C. (2011). Variedades Colombianas de papa. Universidad Nacional de Colombia, p 50. Recuperado de: https://drive.google.com/file/ d/0B0d7qZ6xkUiiRTVzdzd5dnFsdjg/view.

Organización de las Naciones Unidas para la Alimentación y la agricultura (FAO) (2008). Año internacional de la Papa. Recuperado en: http:// www.fao.org/potato-2008/es/index.html.

Organización de las Naciones Unidas para la Alimentación y la agricultura (FAO) (2002). Agua y cultivos logrando el uso óptimo del agua en la agricultura. Recuperado de: http://www.fao.org/ tempref/agl/AGLW/docs/cropsdrops_s.pdf, p 22.

Peña, F., Zenner, I. (2015). Irrigation response of potato (Solanum tuberosum L) var. R12 Diacol capiro. Rev. U.D.C.A Act. \& Div. Cient, 18(2), 385-392.

Porras, P., Herrera, C. (2015)._Modelo productivo de la papa variedad Diacon Capiro para el departamento de Antioquia. Corpoica, Mosquera. 92 p. Recuperado en: https://repository.agrosavia.co/bitstream/ handle/20.500.12324/13751/76688_66357. pdf?sequence $=1 \&$ isAllowed $=y$

Quiroz, R. (2012). Papa o patata. En: Steduto, P.; Hsiao, T.; Fereres, E.; Raes, D. (eds). Respuesta del rendimiento de los cultivos al agua. Organización de las Naciones Unidas para la Alimentación y la agricultura (FAO). Recuperado de: http:// www.fao.org/3/a-i2800s.pdf, p 530.

Reyes-Cabrera, J., Zotarelli, L., Dukes, M., Rowland, D., Sargent, S. (2016). Soil moisture distribution under drip irrigation and seepage for potato production. Agricultural Water Management, (169). Recuperado de: http://dx.doi.org/10.1016/j. agwat.2016.03.001. pp 183-192

Reyes-Cabrera, J., Zotarelli, L., Rowland, D., Dukes, M., Sargent, S. (2014)._Drip as alternative irrigation method for potato in Florida sandy soils. Am. J. Potato Res, 91 (1), 504-516. DOI: 10.1007/ s12230-014-9381-0.
Rodríguez, C., Ruiz de Galerreta, V., Kruse, E. (2015). Analysis of water footprint of potato production in the pampean region of Argentina. Journal of Cleaner Production, (90), 91-96. Doi: http://dx.doi.org/10.1016/j.jclepro.2014.11.075

Rodríguez-Perez, L. (2010). Ecofisiología del cultivo de la papa (Solanum tuberosum L). Rev. Colomb. Cienc. Hortic, 4 (1), 97-108.

Shahnazari, A., Liu, F., Andersen, M., Jacobcen, S., Jensen, C. (2007). Effect of partial root-zone drying on yield, tuber size and water use afficiency in potato under field conditions. Field Crops Research, (100), 117-124. doi: 10.1016/j. fcr.2006.05.010.

Yang, K., Wang, F., Shock, C., Kang, S., Huo, Z., Song, N., Ma, D. (2017). Potato performance as influenced by the proportion of wetted soil volume and nitrogen under drip irrigation with plastic mulch. Agricultural Water Management, (179), 260-270. http://dx.doi.org/10.1016/j. agwat.2016.04.014.

Zhang, Y., Wang, F., Shock, C., Yang, K., Kang, S., Qin, J., Li, S. (2017). Influence of different plastic film mulches and wetted soil percentages on potato grown under drip irrigation. Agricultural Water Management, (180), 160-171. http://dx.doi. org/10.1016/j.agwat.2016.11.018.

Zhou, Z., Plauborg, F., Parsons, D., Andersen, M. (2018). Potato canopy growth, yield and soil water dynamics under different irrigation systems. Agricultural Water Management, (202), 9-18. https://doi.org/10.1016/j.agwat.2018.02.009.

Zin El-Abedin, T., Mattar, M., Alazba, A., Al-Ghobari, H. (2017). Comparative effects of two water-saving irrigation techniques on soil water status, yield, and water use efficiency in potato. Scientia Horticulturae, (225), 525-532. Doi: http://dx.doi. org/10.1016/j.scienta.2017.07.044. 
